\title{
An unusual elbow muscle in the red howler monkey: does it deserve invention of a new name musculus contrahens cubiti?
}

\section{Mellin Novikova and Alexander Kuznetsov}

Faculty of Biology, Lomonosov Moscow State University, Leninskie gory, 1, build. 12, Moscow, 119234, Russian Federation; sasakuzn@mail.ru

Address correspondence and requests for materials to Alexander Kuznetsov

\begin{abstract}
During dissection of a juvenile specimen of howler monkey (Alouatta seniculus), we discovered a supernumerary muscle. This muscle originates from the lateral epicondyle of the humerus, distal to the $m$. brahioradialis and beneath the $m$. extensor carpi radialis longus, and runs deeply to insert on the proximal part of the radius adjacent to the $m$. supinator.

To determine homology of the unusual muscle, we compared it with the known abnormal extra muscles in the group of preaxial forearm extensors in humans and other primates, as well as other mammals. The only similar muscle is the so-called $m$. brachioradialis accessorius, which is very rarely found in humans medial to the $n$. radialis $r$. superficialis. Both in howlers and humans, its unique topological interrelations with the $n$. radialis suggest that this muscle is fundamentally different from all surrounding forearm extensors including the proper $m$. brachioradialis. At the same time, its innervation by the $n$. radialis confirms that it is a true extensor, contrary to the reptilian $m$. tractor radii.

The general problem of identifying homology of anomalies and novelties is considered. As the enigmatic muscle departs from rules of myological architecture of the tetrapod forelimb, we failed to establish its general homology and, instead, suggest naming it as $m$. contrahens cubiti. This means that the muscle acts as the elbow flexor although it intimately belongs to extensors.
\end{abstract}

Keywords: general homology problem, anomaly, forearm extensor muscles, brachioradialis, primates, Alouatta

\section{Introduction}

In this paper, a very unusual forearm muscle will be considered, which was found in one specimen of red howler monkey (Alouatta seniculus) in the course of extensive research on the forearm musculature of the New World monkeys. In mammals, extensor musculature of the forearm and hand originating from the lateral epicondyle of the humerus typically includes: one central muscle - the $m$. extensor digitorum communis; two postaxial muscles - the $m$. extensor digitorum lateralis and $m$. extensor carpi ulnaris; and four preaxial muscles - the $m$. supinator, $m$. brachioradialis (termed in the old literature $m$. supinator bre$v i$ and $m$. supinator longus, respectively), m. extensor carpi radialis longus, and $m$. extensor carpi radialis brevis. In our specimen of the howler monkey, the fifth preaxial forearm extensor was present bilaterally.

Anatomical abnormalities rarely occur in the wild, especially rare are new formations, and even more rarely they are captured by anatomists. Because of data scarcity, the theoretical basis for analysis of natural anatomical anomalies is poorly developed, and to properly present the morphological significance of our finding, we have to formulate the principles ourselves, according to fundamentals of comparative anatomy. Among few useful publications on the topic is "The

Competing interests: The authors have declared that no competing interests exist. 
logic of monsters..." by Pere Alberch (1989), the founder of Evo-Devo. But its dashing title is rather misleading for understanding abnormalities, because it seemingly presumes that monsters bear some logic of their own, which is independent of the logic of normally developed organisms. This presumption is incongruous, as monsters are not united with each other directly, bypassing the normal organisms. Undoubtedly, "monsters" and anomalies (i.e. partial monsters) are the sprouts of the logic of norm. So, by studying anomalies, we study the general logic of the morphological architecture, its degrees of freedom and constraints.

When an anomaly is identified, a name should be given, like in the case of normal organs. To avoid excess multiplication of terms, the anatomist investigates the literature in search of previous findings of the most similar anomalies within the same species, or around the taxonomic neighborhood. If no precedent is found, a new appropriate term is invented. Since Joseph Maclise (1846) and Richard Owen (1846) invented the terms "archetype" and "homology", in the anatomical thesaurus, anatomists avoid using the same names for non-homologous structures. It is more difficult to identify homologies of abnormal structures than of normal ones. The problem is that, due to the rarity of abnormalities, it is usually technically impossible, to approve that two anomalies labeled with the same term bear the same genetic basis. Does this mean that every case of anomaly with unknown genesis should be cautiously termed with a unique name? Or, per contra, anomalies do not deserve to be termed at all? Or, may be only heritable anomalies deserve proper names? Actually, there is often no confidence in whether the anomaly is a heritable trait or a non-heredity morphosis (sensu Schmalhausen, see Levit et al., 2006). For Richard Owen, when he began to introduce the concept of archetype and homology into anatomical practice (Owen, 1848, 1849), these questions were much easier to solve, than to modern biologists. This is the penalty for Darwin's suggestion to incorporate Owen's ideas into evolutionary theory by straightforward equalization of his archetype with an ancestor; since then, homologization is virtually prohibited without the knowledge of heredity and phylogenetic origin. To loosen the fetters and bypass the difficulties, which derive from equalization of archetype to ancestor, a tremendous amount of shades of homology were noticed and termed (Pavlinov, 2012). In our case, this terminological activity fails to help make a step forward in the simple applied question of how to treat the unusual howler's muscle. It is thus more useful to rely on Owen.

The kernel of Owen's concept of homology (the structural unity) as opposed to analogy (functional unity) was composed of two structural diagrams and three linking notions. The structural diagrams are (i) the ideal typical vertebra representing the elementary building block of the vertebrate body and (ii) the archetype depicting the normal composition (linear series) of these elementary building blocks. The linking notions are (i) the general homology, (ii) special homology, and (iii) serial homology. The priority of terms "archetype" and "homology" was contested by Maclise (Rupke, 1993), and the vertebrate archetype diagram was definitely developed by Owen on the base of similar diagrams published by Carl Carus 20 years earlier (Rupke, 1993; Richards, 2016). Thus, the major novelty of Owen himself was the demarcation of three sorts of homology, of which the first and most important has been almost entirely lost by contemporary biology. It is the general homology, which was Owen's tool to associate, part by part, real organisms with the ideal archetype. As soon as Darwinism kidnapped the archetype concept and equalized it with the ancestral form, the necessity in the concept of general homology vanished, because the ancestor, even if unknown to science, was definitely built from the same matter as its offsprings, and, hence, the special homology is adequate to associate its body parts with theirs. In the process of substitution of the archetype with the ancestor, nobody noticed an arising absurdity: as an organism has an innumerable chain of ancestors, it is obviously senseless to adopt every one of them as a new archetype. But if we decide that not every ancestor deserves the rank of archetype, the question arises of how to distinguish those which deserve? Owen did not face such an artificial problem, because he did not expect that Nature bears numerous archetypes. More than that, the less is the number of archetypes, to which the diversity of real bodies can be reduced, the greater is, hence, the efficiency of comparative anatomy. The archetype in anatomy, like laws in physics, is the triumph of generalization. Owen repeatedly emphasized, that the general homology of organs of a real organism is established by naming those in terms of the archetype. Implicitly, we often follow the same procedure today. For instance, when comparing the hindlimb muscles of real tetrapods, we state the special homology of mammalian $m$. obturator externus and avian $m$. obturator internus, while the general homologization with the myological archetype of the tetrapod hindlimb is performed when we term both of these special muscles as the m. puboischiofemoralis externus. Only those real organisms belong to particular archetype, which organs and structures can be entirely named in terms of this archetype. If an organ is found, which does not fit there, this means that its general homology cannot be identified and, hence, an organism with this organ belongs to some other archetype, may be not yet defined.

Ironically, the organs, to which Owen devoted a special writing (Owen, 1849) - the paired limbs of vertebrates - cannot be mapped to the archetype he invented. Indeed, the paired limbs of vertebrates, contrary 
to those of arthropods, are not the accessories of singular body segments, but represent supersegmental compositions. Owen should not have overlooked their complex design being well aware of the fact that every nervous plexus of the pectoral and pelvic limb is formed by a series of spinal nerves. But he seems to have been so much blinded by the beauty of segmentalist designs of Goethe, Carus, and his own, that he dared to homologize each limb girdle with a single rib, and the free limb skeleton with its uncinate process. In fact, if Owen's archetype is applicable at all, it is only applicable to agnathan vertebrates and lower chordates which lack paired limbs. The evolutionary appearance of these appendages marked the generation of a new vertebrate archetype - the body with supersegmental organs, which have no name in terms of general homology with parts of the previous archetype. The paired limbs represent novel extensions of the vertebrate morphospace with their own morphogenetic degrees of freedom and constraints which restrict the possible diversity of anomalies.

We shall analyze the discovered unusual muscle of the red howler monkey from Owen's classic standpoint as the most consistent one. In order to establish the muscle's general homology, that is to map it in coordinates within the general structural plan of the forearm musculature of tetrapods, we have to analyze morphogenetic degrees of freedom of this body region with the help of available data on structural variations of all adjacent muscles, namely, the $m$. supinator, $m$. brachioradialis, $m$. extensor carpi radialis longus, and $m$. extensor carpi radialis brevis. Most probably, we may deal with a duplicate muscle, as doubling is the most common kind of morphological anomalies (Alberch, 1989). If the abnormal muscle is not a simple duplicate of one of its neighbors, then we should check if it fits into series of homologous variations sensu Vavilov (1922), known in the musculature of other species (first, in the closer relatives of howler monkeys, then in more phylogenetically distant forms). Further, we should consider the abnormal muscle in the view of possible atavistic recapitulation of some ancestral muscle. If one or the other way of analysis leads to successful establishment of the muscle's general homology, we will provide an appropriate name from the standard myological nomenclature. However, if the anomaly under consideration does not fit within the known archetype of the tetrapod limb, we shall introduce a new term for it.

\section{Materials and methods}

Dissection of the forearm muscles was undertaken in 9 platyrrhine species: Venezuelan red howler Alouatta seniculus Linnaeus, 1766 (1 juv), bald uakari Cacajao calvus I.Geoffroy, 1847 (1 juv), white-headed capuchin Cebus capucinus Linnaeus, 1758 ( 1 ad), tufted ca- puchin Sapajus apella Linnaeus, 1758 (1 ad), common squirrel monkey Saimiri sciureus Linnaeus, 1758 (1 juv, $1 \mathrm{ad})$, common marmoset Callithrix jacchus Linnaeus, 1758 (2 ad), black-tufted marmoset Callithrix penicillata É. Geoffroy, 1812 (1 ad), pygmy marmoset Cebuella pygmaea Spix, 1823 (1 ad), and golden lion tamarin Leontopithecus rosalia Linnaeus, 1766 ( $1 \mathrm{ad})$. One specimen of green monkey Chlorocebus sabaeus Linnaeus, 1758 ( 1 juv) was also dissected as representative of outgroup (catarrhines). The material for dissection fixed in formalin or alcohol was granted by the Department of Vertebrate Zoology and the Zoological Museum of Moscow State University and the Zoological Institute of Russian Academy of Sciences, Saint Petersburg; Alouatta seniculus possessing the unusual muscle belongs to the Zoological Institute. Manipulations were performed using a stereo microscope Carl Zeiss Stemi SV 11. All stages of dissection were filmed with a digital camera Canon PC1562. For each species detailed descriptions of the muscles topography including their relations with nerves as well as detailed anatomical drawings were made. The nomenclature of muscles, bones and nerves, which is used here, follows human anatomy. If necessary, old synonyms are referred to, as well as some special names for anomalies and non-mammalian muscles. In descriptions of muscles, the proximal attachment is always regarded as the origin, and the distal one as the insertion. When muscle portions have separate origins but a single insertion, they are treated as heads; the opposite case is termed bifurcation.

\section{Results}

In all studied primates, there was a full set of the extensor muscles of the forearm and hand originating from the lateral epicondyle of the humerus: the $m$. supinator, $m$. brachioradialis, $m$. extensor carpi radialis longus, $m$. extensor carpi radialis brevis, $m$. extensor digitorum communis, $m$. extensor digitorum lateralis, and $m$. extensor carpi ulnaris. All these muscles were supplied by the deep branch of the radial nerve (n. radialis r. profundus). This is consistent with data from other researchers, as reviewed by Diogo and Wood (2012). Of four preaxial forearm extensors, the $m$. supinator was entirely hidden below the others, which are immediately visible under the skin on the forearm surface in the following anteroposterior order: $m$. brachioradialis, $m$. extensor carpi radialis longus, $m$. extensor carpi radialis brevis.

Besides the above mentioned set of muscles, we further found, in both forelimbs of the single juvenile specimen of the red howler monkey (Alouatta seniculus), which was at our disposal, an additional elbow muscle descending obliquely from the humerus to the radius (Fig. 1A-C). Like all the forearm extensor muscles, it was also innervated by the $n$. radialis $r$. profun- 

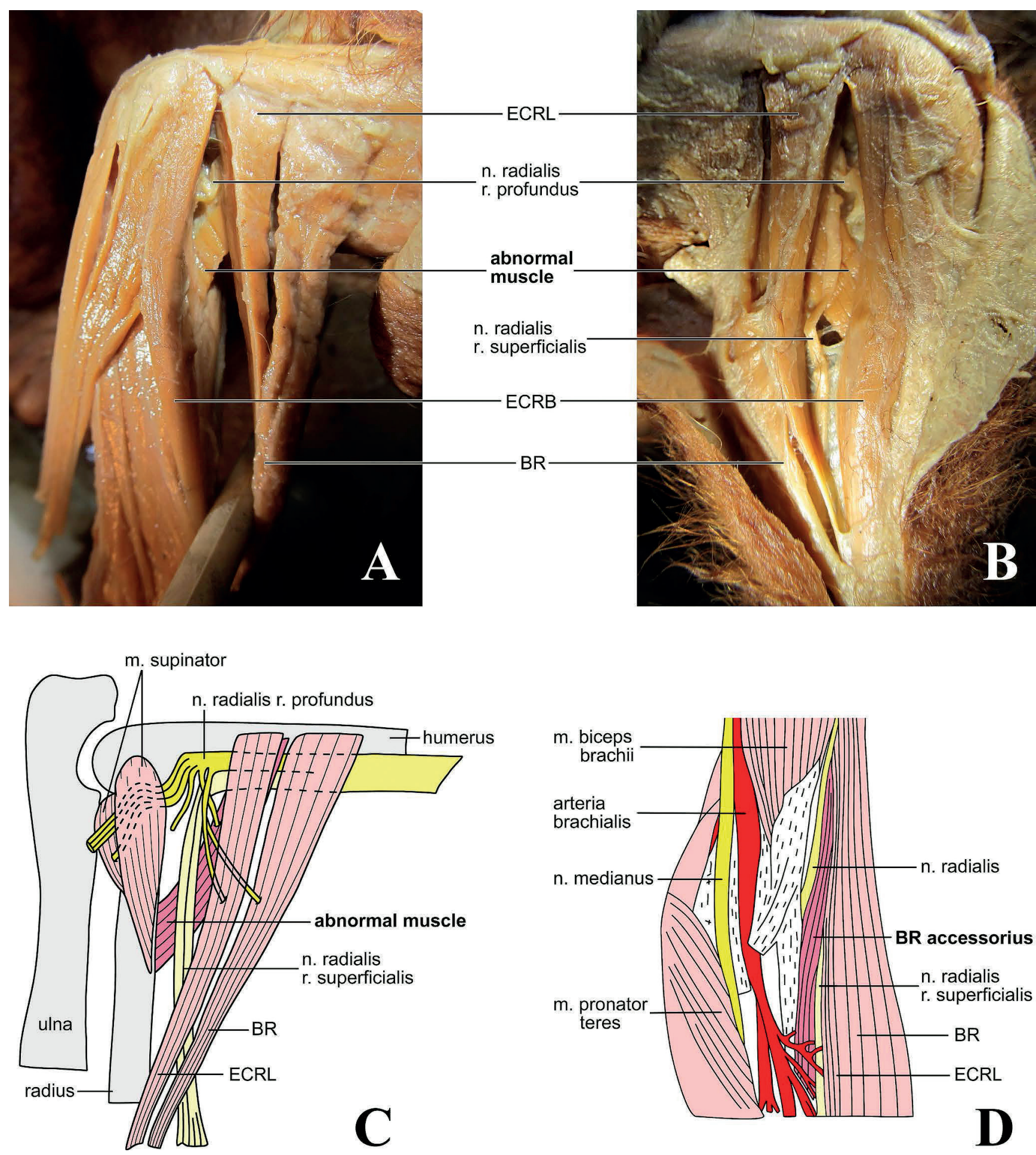

Fig. 1. Lateral view of preaxial extensor muscles of the (A) right and (B) left forearm of Alouatta seniculus; $\mathbf{C}$ - schematic drawing of the muscles and nerves corresponding to (A); D - palmar (anterior) view of the left human elbow with a similar muscle (redrawn from Rodriguez-Nedenführ et al., 2001). BR - m. brachioradialis, ECRL $-m$. extensor carpi radialis longus, ECRB - m. extensor carpi radialis brevis.

dus. This fifth preaxial muscle was located under the $m m$. extensores carpi radiales. Its rather short and thick parallel-fibred belly had a fleshy origin on the lateral epicondylar crest immediately anterior to the $m$. extensor carpi radialis longus and a fleshy insertion on the lateral surface of the radius immediately anterior to that of the m. supinator. So, together with the latter, the fifth muscle formed the deep layer of preaxial forearm extensors. However, its apparent action is very different from that of the $m$. supinator: judging by its position, it should flex the elbow, rather than supinate the radius relative to the ulna. The second important difference from the m. supi- 
nator is that the supernumerary muscle passed lateral to the $n$. radialis stem before its branching. More distally, it traversed medial to the $n$. radialis $r$. superficialis, in contrast to the $m$. brachioradialis and $m m$. extensores carpi radiales. We did not find in other monkeys, which we dissected, any trace of such supernumerary muscle as in the howler.

\section{Discussion}

In search for homologues of the abnormal howler's muscle, let us consider the known diversity of the other four preaxial forearm extensors, namely the $m$. supinator, $m$. brachioradialis, $m$. extensor carpi radialis longus, and $m$. extensor carpi radialis brevis. This diversity includes, on one hand, interspecific variations and, on the other hand, individual abnormalities. Since the full set of the four normal muscles was present in our howler monkey specimen, we will take into account only the cases with supernumerary muscular units, such as duplications, additional heads, and bifurcations, generally referred to as splitting.

\section{Splitting of the m. supinator}

Typically, this muscle is composed of one or two heads. If two, they are separated from each other by the $n$. radialis $r$. profundus (which then passes into the $n$. interosseus antebrachii dorsalis), the external head originating from the lateral epicondyle of the humerus, whereas the internal head arises from the elbow joint capsule, or even from the ulna, according to Diogo and Wood (2012).

The overview of human variations of this muscle is presented in the online Illustrated Encyclopedia of Human Anatomic Variation (Bergman et al., 2000). In general, the diversity of these variations is smaller than in the other preaxial extensors of the forearm (see below) being restricted to various degrees of subdivision of the muscular belly by the $n$. radialis $r$. profundus. Normally, humans have a single belly, and the $n$. radialis r. profundus passes through its base under the so-called "arcade of Frohse", represented by a ligamentous bridge from which muscular fibers of the m. supinator arise. As a variation, the belly may be cut by a longitudinal cleft for the nerve passage, and in the extreme case this cleft entirely divides the belly into two separate heads. This case is sometimes treated as doubling of the muscle, instead of subdivision, and the head, which is deeper to the nerve, has been sometimes specially termed, as m. supinator accessorius (LeDouble, 1897). Bergman et al. (2000) have mentioned the case, where this head originated from the lower border of the $m$. brachialis, which is interesting as an example of generally rare associations of extensor and flexor muscles of the limbs. Bergman et al. (2000) summarize that the separate deep head has been found in 21 of 63 human forearms, and bilaterally in 8 of 27 cadavers.
In a number of non-human mammals, including the New World monkeys, except of the marmosets, it is normal that the deep head of the $m$. supinator is separate from the superficial one. In our howler monkey, the two heads of the $m$. supinator were also separate. Although they converged onto the anterolateral surface of the radius, along with the abnormal muscle in question, the latter one originated from the humerus too far proximally (Fig. 1A-C) to be regarded as a third head of the $m$. supinator, which is unknown elsewhere. Also against affinity with the $m$. supinator is their aforementioned sharp functional difference: judging by its position, the howler's supernumerary muscle mainly flexes the elbow, while the $m$. supinator supinates the radius relative to the ulna.

Splitting of the $m$. brachioradialis (Fig. 2)

The duplication of the $m$. brachioradialis (Fig. 2A) is regarded as a very rare human abnormality observed in $0.5-1 \%$ of individuals (Carrington et al., 1883 and Gruber, 1848 in Rodriguez-Nedenführ et al., 2001), but according to recent data (Rodriguez-Nedenführ et al., 2001), the twin $m$. brachioradialis is found in $2.8 \%$ of humans. Albright and Linburg (1978) have mentioned cases of duplication of the $m$. brachioradialis not only in humans but in other mammals too. They named the additional muscle " $m$. extensor carpi radialis accessori$u s$ ", although it is definitely the twin of $m$. brachioradialis, as its tendon inserts on the distal end of the radius, and not on the metacarpus, as the $\mathrm{mm}$. extensores carpi radiales do.

In humans, there is also a special case of duplication of the $m$. brachioradialis (Fig. 2B) first described by Kaneff (1969, cited in Claassen and Wree, 2002). The accessory muscle in question originated on the lateral epicondyle between the normal $m$. brachioradialis and $m$. extensor carpi radialis longus and inserted on the os trapezium of the carpus. (Sometimes, the $m$. brachioradialis inserts even more distally: Wood (1867) described its bifurcation with an additional insertion on metacarpal I (Fig. 2C).

The origin of additional head of $m$. brachioradialis can be shifted far proximally along the humerus (Fig. 2D). In humans, such proximal origin of additional head has been found near the insertion of $m$. deltoideus (Gruber, 1848, cited after Rodriguez-Nedenführ et al., 2001). Dunlap et al. (1985) described a similar additional head of the $m$. brachioradialis in squirrel monkeys (Saimiri sciureus): its origin was found on the pectoral crest of the humerus, medial to the insertion of the $m$. pectoralis major (s. superficialis). Apparently, the presence of two heads of the $m$. brachioradialis, one running from the lateral epicondyle and the other from the pectoral crest, is the usual condition in squirrel monkeys. We also observed them in both forelimbs of our squirrel monkey specimen; they converged from the lateral epi- 


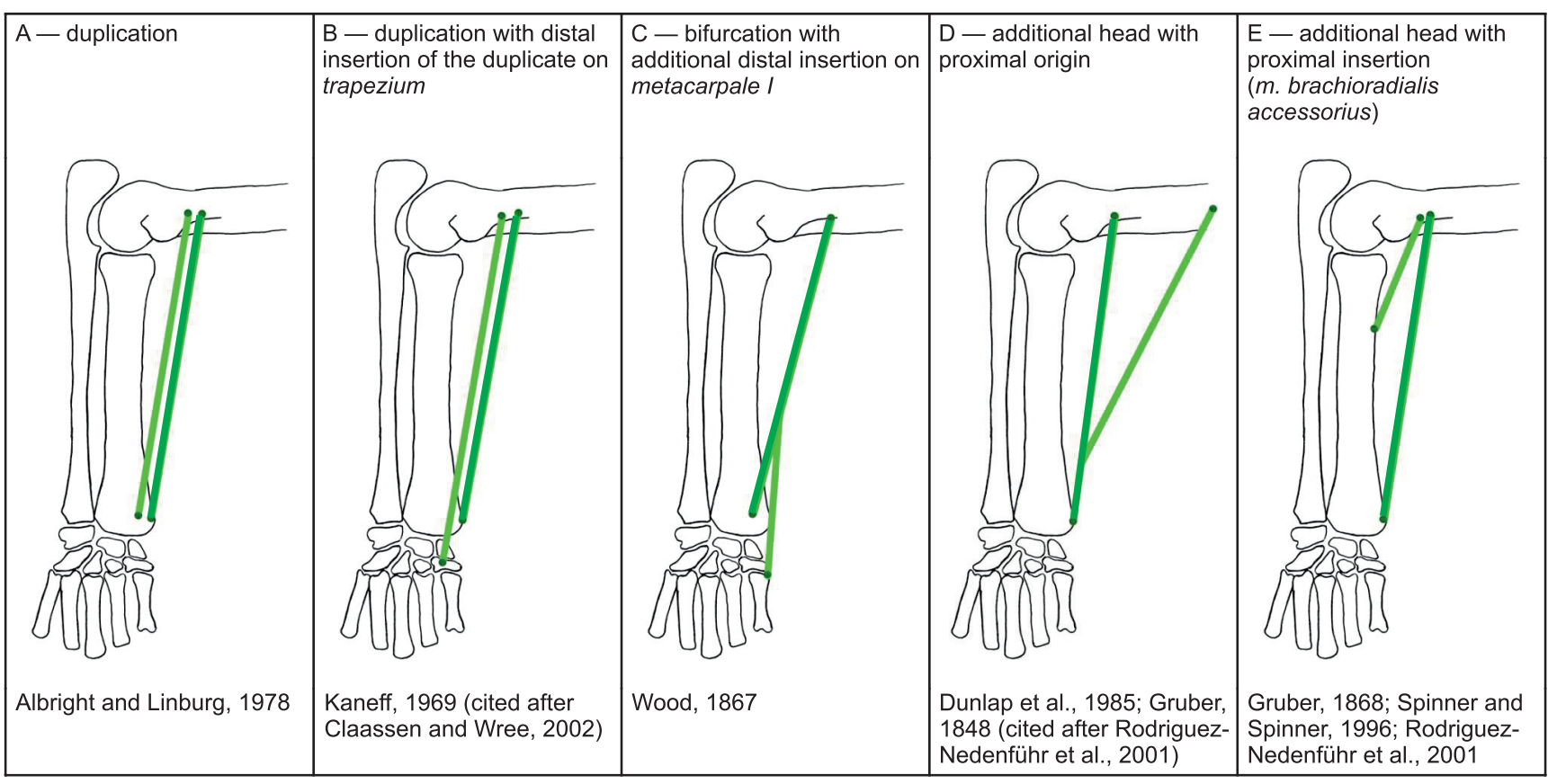

Fig. 2. Graphic summary of the known splitting variations of the mammalian $m$. brachioradialis (mainly based on data from human anatomy). Green — the typical muscle, light green - its variations.

condyle and the pectoral crest and fused together at the distal epiphysis of the radius.

Alternatively, the additional $m$. brachioradialis originates from the lateral epicondylar crest near the original one (even a little more distally), but there is a pronounced proximal shift of its insertion along the radius (Fig. 2E). Such a muscle has been reported as a rare human variation by several authors and has received several names (remember that the old synonym of the normal $m$. brachioradialis is $m$. supinator longus): Gruber (1868) called it $m$. brachioradialis minor $s$. brevis, and also $m$. supinator longus II (in this case he called the normal $m$. brachioradialis as the $m$. supinator longus I); Spinner and Spinner (1996) termed it $m$. brachioradialis brevis or $m$. supinator longus accessorius; the latter name used earlier by Lauth (1830, cited in Bergman et al., 2000); Rodriguez-Nedenführ et al. (2001) described it as $m$. brachioradialis accessorius. This abnormal human muscle usually inserts near midlength of the radius (LeDouble, 1897). More precisely, Gruber (1868) reported its insertion on the radial tuberosity and on the terminal tendon of the m. biceps brachii, sometimes on the terminal tendon of the $m$. supinator. According to Rodriguez-Nedenführ et al. (2001), it inserted on the radius very proximally - on its anterolateral surface ( 2 cases), together with the $m$. biceps brachii ( 2 cases, one of which is reproduced in our Fig. 1D), or together with the $m$. pronator teres ( 1 case). Considering the general position and, hence, function and its topological interrelations with the $n$. radialis (proximally, the muscle is lateral to the nerve, while, distally, they swap), this abnormal $m$. brachioradialis accessorius in humans appears most similar to the supernumerary muscle we have identified in our howler monkey specimen.

Notably, in gibbons, $m$. brachioradialis inserts much more proximally than usual, closer to midlength of the radius (Diogo and Wood, 2012), and thus, also appears to be somewhat similar to the abnormal muscle of our howler monkey specimen and the human $m$. brachioradialis accessorius. However, it is more parsimonious to assume that the muscle of gibbons is the proper $\mathrm{m}$. brachioradialis shifted proximally due to extreme forearm elongation, than that the proper muscle is lost, and the unusual $\mathrm{m}$. brachioradialis accessorius has become the norm instead. Wood (1867) had claimed that he found the homologue of $m$. brachioradialis accessorius among the normal muscles of the platypus (Ornithorhynchus) and short-beaked echidna (Tachyglossus). Westling (1889), for the short-beaked echidna, and Allen (1912), for the longbeaked echidna (Zaglossus), regarded the same muscle as the proper $m$. brachioradialis. In the most recent redescription covering all three monotreme genera, this muscle is identified as the radial head of the common elbow flexor of all tetrapods, the $m$. brachialis, and is specially termed as $m$. brachialis radialis (Gambaryan et al., 2015). It has flexor innervation, and we cannot exclude the suggestion of Haines (1939) that this monotreme muscle is homologous to the ancestral $m$. tractor radii (vide infra).

Splitting of the mm. extensores carpi radiales (Figs 3-5)

Most variable of all preaxial forearm extensors are the two mm. extensores carpi radiales. Normally, in most 


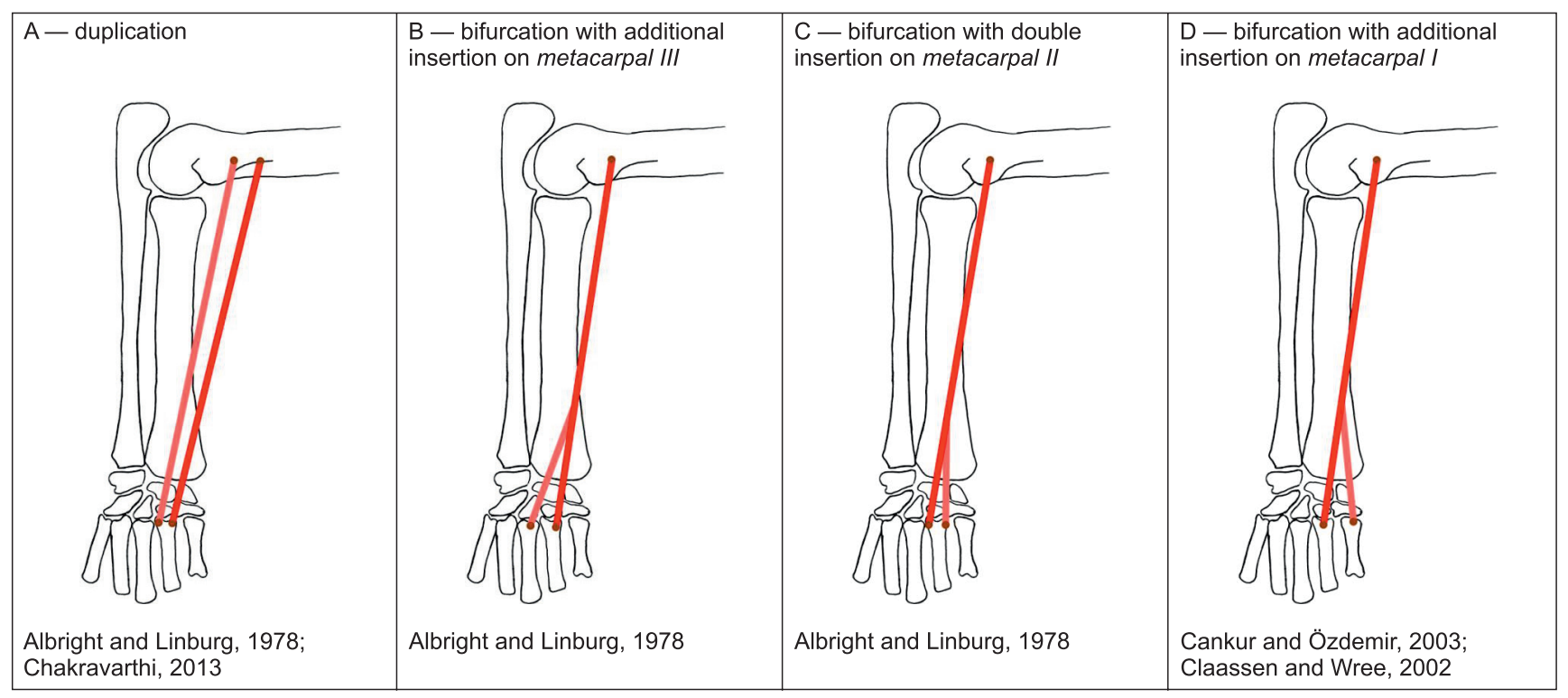

Fig. 3. Graphic summary of splitting variations of $m$. extensor carpi radialis longus known in mammals (mainly based on data from human anatomy). Red - the typical muscle, light red - its variations.

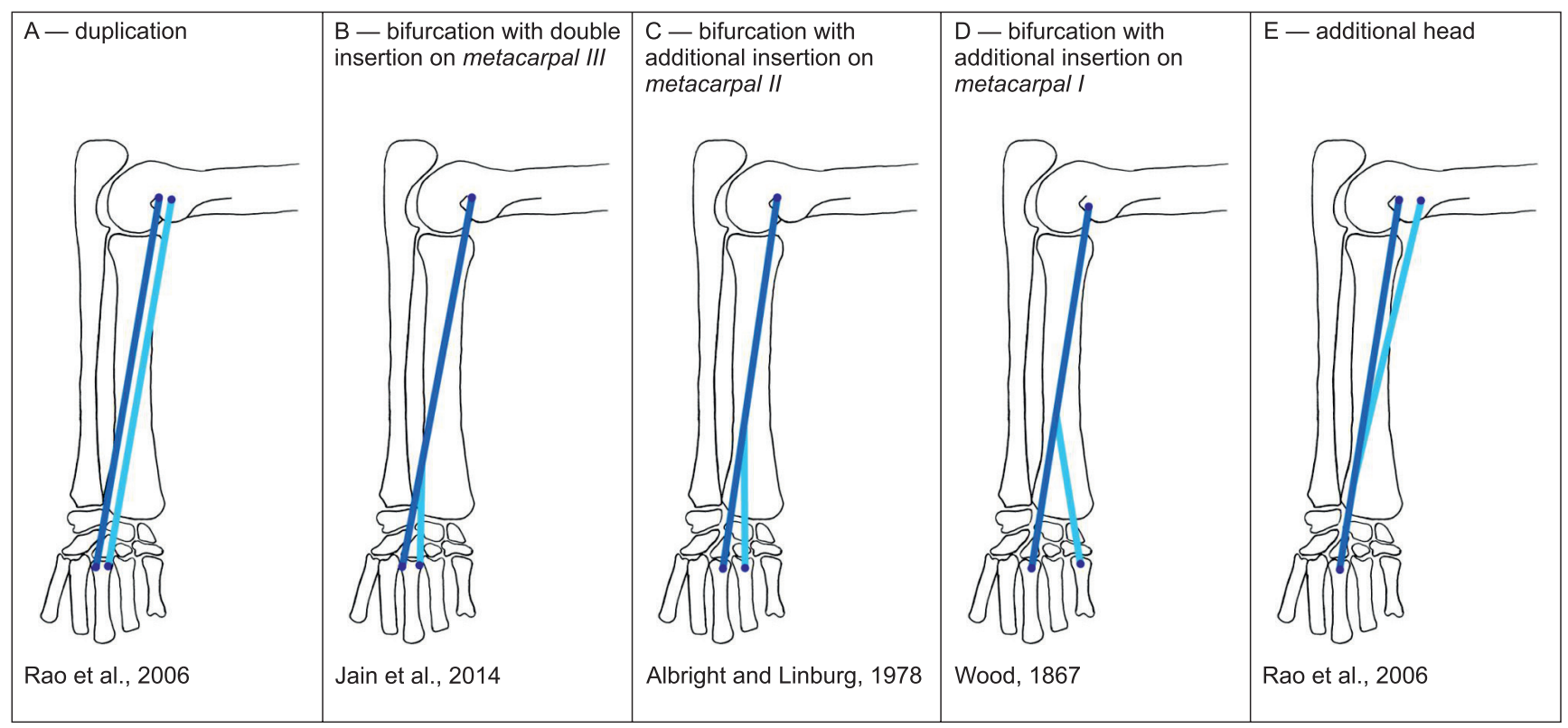

Fig. 4. Graphic summary of splitting variations of $m$. extensor carpi radialis brevis known in mammals (mainly based on data from human anatomy). Blue - the typical muscle, light blue - its variations.

primates including humans, they are quite separate from each other and descend from the lateral epicondyle side by side - the $m$. extensor carpi radialis longus is more anterior and inserts at the base of the metacarpal II, while the m. extensor carpi radialis brevis is more posterior and inserts at the base of the metacarpal III. However, it is convenient to consider their variations together, insofar as, during embryonic development, they both differentiate from a single anlage (Albright and Linburg, 1978; Cankur and Özdemir, 2003). Their unity is sometimes retained even in the adult stage: a human anomaly is known (Wood, 1867), where the terminal tendons to metacarpals II and III (which can be split into up to 5 tendinous branches) derive from a single belly representing both $m m$. extensores carpi radiales together.

Variations in these muscles are well known in humans due to their particular interest associated with the use of their tendons in hand surgery including tendon transfer, thumb opposition, surgical rehabilitation of patients with paralytic disorders, etc. (Wood, 1988; Khaledpour and Schindelmeiser, 1994; Cankur and Özdemir, 2003; Rao et al., 2006; Nayak, 2008). The presence 


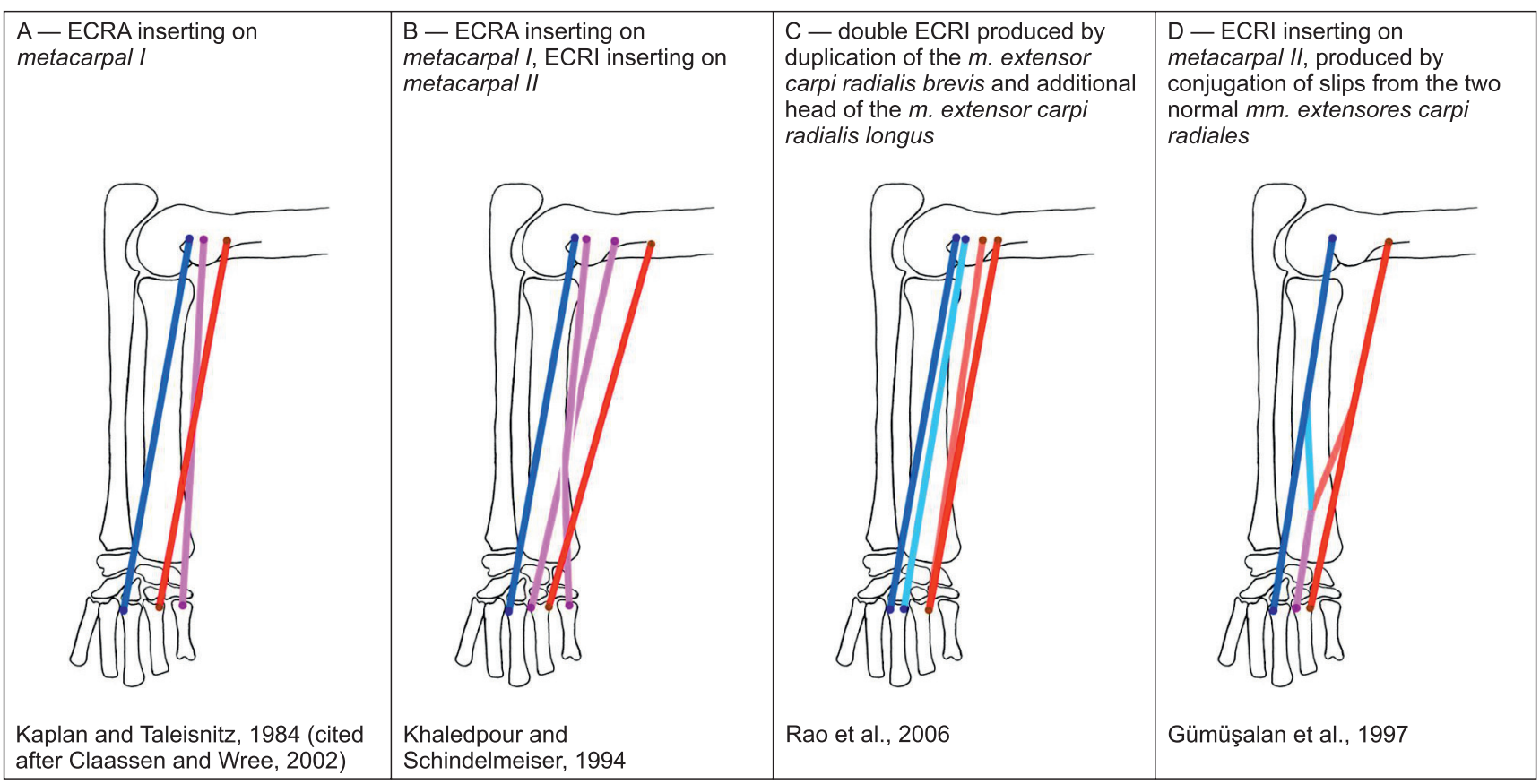

Fig. 5. Graphic summary of additional $m m$. extensores carpi radiales known in mammals (mainly based on data from human anatomy). Magenta $-m$. extensor carpi radialis accessorius (ECRA) and $m$. extensor carpi radialis intermedius (ECRI) sensu Wood, 1867; other colors as on Figs $3,4$.

of duplication of muscle, or additional head or terminal tendon has been reported in $12-50 \%$ of individuals, the additional structures being often found in both arms (Albright and Linburg, 1978; Khaledpour and Schindelmeiser, 1994; Gümüşalan et al, 1997; Claassen and Wree, 2002; Cankur and Özdemir, 2003; Hong and Hong, 2005; Rao et al. 2006; Nayak et al., 2008; Chakravarthi, 2013).

More frequent, in humans, are various bifurcations. The proximal bifurcation looks like an additional slip, which splits off from the upper part of the belly of the $m$. extensor carpi radialis longus or $m$. extensor carpi radialis brevis, while the distal bifurcation looks like an additional tendinous branch deviating from the normal terminal tendon of one of these muscles and inserting on metacarpal I, II or III (Figs 3,4). There is a case where the $m$. extensor carpi radialis longus sends a fiber bundle joining the $m$. brachioradialis (Wood, 1867). Bifurcated tendons of the $m$. extensor carpi radialis brevis with insertions on metacarpals II and III have been also reported as the normal condition in some capuchins (Hill, 1960) and gibbons (Michilsens et al., 2009 cited in Diogo and Wood, 2012).

More rarely found are supernumerary muscular heads of the radial carpal extensor complex, which originate from the humerus (the lateral epicondyle) separately from the normal two heads (i.e., the m. extensor carpi radialis longus and $m$. extensor carpi radialis brevis). Wood (1867) has designated the two most common supernumerary muscles in humans, as $m$. extensor carpi radialis intermedius and $m$. extensor carpi ra- dialis accessorius (Fig. 5). They may be present together (Fig. 5B) and are distinguished from each other by their insertion points: $m$. extensor carpi radialis intermedius inserts on metacarpals II or III, like a twin of m. extensor carpi radialis longus or $m$. extensor carpi radialis brevis, respectively; on the other hand, $m$. extensor carpi radialis accessorius looks more unusual inserting on metacarpal I. Both of these additional muscles are quite variable (Fig. 5), but they never insert on the radius, even at its distal tip, and in this respect sharply differ from the supernumerary muscle of our howler monkey specimen.

Summarizing the available literature, we can state that a similar muscle has not been reported neither as the normal condition, nor as an anomaly in either howlers (Sirena, 1871; Schön, 1968; Youlatos, 1999), or in other platyrrhines (Dunlap et al., 1985; Youlatos, 2000), or even in non-human primates as a whole (Diogo and Wood, 2012). Additionally, never have we met a single reference of this muscle in any non-primate mammal, and nothing whatsoever in the recent review by Diogo and Abdala (2010).

We conclude that the supernumerary muscle, which we have identified in our juvenile red howler specimen, can be most likely interpreted as the homologous variation of the abnormal human $m$. brachioradialis accessorius, in the sense of Vavilov's "law of homologous series in variation" (Vavilov, 1922). However, even in humans, it is still uncertain if this supernumerary muscle is really a sprout of the $m$. brachioradialis or something fundamentally separate. What further complicates is the 
fact that two topologically different human anomalies have been reported under the name " $m$. brachioradialis accessorius" (Spinner and Spinner, 1996). Both reported abnormal muscles looked similar in relation to the surrounding muscles and bones, but their position was different relative to the $n$. radialis $r$. superficialis. In some cases the short additional brachioradial muscle was found lateral to this nerve, like the normal $\mathrm{m}$. brachioradialis (Spinner and Spinner, 1996). In other cases the externally similar supernumerary muscle passed medially, so that the same nerve separated it from the normal $m$. brachioradialis (Rodriguez-Nedenführ et al., 2001) (Fig. 1D). Our howler monkey specimen showed the second variant, which looks more surprising in the framework of normal topology of surrounding muscles and nerves. Indeed, the diagram in Fig. 1C clearly shows that this variant of abnormal muscle could not arise from any of the surrounding muscles without overpassing one or another branch of the $n$. radialis: the internal head of the $m$. supinator should overpass laterally by its origin, while the other muscles should overpass medially by their insertions. The idea that the overpass was performed by a sprout of the $m$. brachioradialis, rather than other muscles, is favored by another kind of human anomaly. In this case, the $n$. radialis $r$. superficialis pierces between slips of the split terminal tendon of the $m$. brachioradialis, as if the medial slip of this tendon has overpassed the nerve (Bergman et al., 2000; Shetty et al., 2014). This implies that the anlage of the $m$. brachioradialis possesses a morphogenetic degree of freedom to spread medial to the embryonic route of this nerve.

Perhaps, the search for homologues of the $\mathrm{m}$. brachioradialis accessorius, both in howlers and humans, should not be restricted to mammals, which, to our knowledge, lack it at all (e.g. Diogo and Abdala, 2010). In a review of the extensor muscles of the forelimbs of tetrapods, Haines (1939) drew attention to an unusual muscle, which he called the $m$. tractor radii. In chelonians, Haines distinguished four preaxial extensors in the forearm. Three of them seem to be homologous to mammalian muscles as follows:

$m$. extensor radialis profundus $=m$. supinator,

$m$. extensor radialis intermedius $=m$. brachioradialis,

m. extensor radialis superficialis $=m$. extensor carpi radialis longus + brevis

$m$. tractor radii $=$ ???

If, indeed, the third chelonian extensor corresponds to two muscles of the mammalian set, then the fourth chelonian muscle, which is the $m$. tractor radii visible immediately under the skin, could be a homologue of some fifth one - why not of the so-called $m$. brachioradialis accessorius of humans and, eventually, howlers? But, in sharp contrast with the other three mentioned muscles, which are innervated by the $n$. radialis (Haines called it the $n$. extensorius cranialis), the $m$. tractor radi $i$, is innervated by a special branch from the nerves supplying flexor muscles (of which, most closely placed to the forearm extensors is the $m$. brachialis). According to Haines (1939), the tuatara and monitor lizards also possess the $m$. tractor radii. He regarded this muscle as a serial homologue to the preaxial head of the gastrocnemius in the hindlimb. Haines hypothesized, that the $m$. tractor radii would be present in the original muscular set of primitive tetrapods such as Eryops, and that in modern amphibians, crocodiles, and mammals it has secondarily disappeared together with the corresponding nerve branch or, which seemed to Haines less likely, fused into the $m$. brachioradialis ( $m$. extensor radialis intermedius of Haines). Haines even suggested the homologue for the $m$. tractor radii in monotremes (based on description by Westling, 1889). If Haines is right, then the $m$. tractor radii was present in the evolutionary history of mammals and may be found in some of them as a rare atavistic anomaly. So, if homology between the howler's supernumerary muscle and $m$. tractor radii was proven, Haines' (1939) hypothesis on the presence of $m$. tractor radii in the archetype of all tetrapods and the atavistic nature of this anomaly would be validated. However, this hypothesis is immediately falsified. The crucial difference of the $m$. tractor radii from all other muscles originating from the lateral epicondyle of the humerus is the nerve supply. Apparently, the flexor-like innervation of the $m$. tractor radii completely excludes the possibility that the $m$. brachioradialis accessorius of howlers and humans, supplied by the $n$. radialis $r$. profundus, is an atavistic $m$. tractor radii. In the same way, the extensor innervation of the $m$. brachioradialis accessorius precludes the idea that it has split off from the adjacent $m$. brachialis or $m$. biceps brachii, the main elbow flexors running along the humerus.

\section{Conclusion}

It should be emphasized that the enigmatic muscle described in this work was discovered in a juvenile red howler specimen, in which all typical forearm extensors running from the lateral epicondyle were well developed and typically placed. Among all known variations of preaxial forearm extensors, this muscle looks most similar to the medial variant of the $m$. brachioradialis accessorius, which is rarely found in humans and, until now, nowhere else. Similar to the normal $m$. brachioradialis, the supernumerary counterpart is able to participate in elbow flexion, despite of the extensor innervation and grouping. However, its unique topological interrelations with the $n$. radialis imply its fundamental difference from the normal $m$. brachioradialis. Its origin remains uncertain but, in any case, it could not arise from the flexor muscles such as the $m$. brachialis, $m$. biceps brachii, or the archaic $m$. tractor radii retained in chelonians, tuataras, monitor 
lizards, and, probably, monotremes. Regarding its unique morphology, it may be worthy giving this muscle a new, function-based, name: "musculus contrahens cubiti".

In all cases, further research in more howler monkey specimens, as well as other platyrrhines is necessary to decide, whether $m$. contrahens cubiti is a common feature or a very rare abnormality.

\section{Acknowledgements}

We would like to acknowledge Aleksandra A. Panyutina for valuable advice. The help of anonymous reviewer on English language improvement was invaluable.

\section{References}

Alberch, P. 1989. The logic of monsters: evidence for internal constraint in development and evolution. Geobios 22:21-57.

Albright, J.A., and Linburg, R. M. 1978. Common variations of the radial wrist extensors. Journal of Hand Surgery 3:134-138.

Allen, G. M. 1912. Zaglossus. Memoirs of the Museum of Comparative Zoology, Harvard College 40:253-307.

Bergman, R. A., Afifi, A. K, and Miyauchi, R. 2000. Illustrated Encyclopedia of Human Anatomic Variation. Part I: Muscular System. http://www. anatomyatlases.org/AnatomicVariants/AnatomyHP. shtml

Cankur, N.Ş., and Özdemir, S.T. 2003. Accessory slip of the extensor carpi radialis longus muscle. Gazi Medical Journal 14:197-199.

Chakravarthi, K. K. 2013. An additional extensor carpi radialis longus muscle and its clinical significance. International Journal of Bioassays 2:887-888.

Claassen, H., and Wree, A. 2002. Multiple variations in the region of $\mathrm{mm}$. extensores carpi radialis longus and brevis. Annals of Anatomy 754:489-91.

Diogo, R., and Abdala, V. 2010. Muscles of Vertebrates: Comparative Anatomy, Evolution, Homologies and Development. Science Publishers, Enfield (New Hampshire).

Diogo, R., and Wood, B. 2012. Comparative Anatomy and Phylogeny of Primate Muscles and Human Evolution. Taylor \& Francis, Oxford.

Dunlap, S. S., Thorington, R. W. Jr., and Aziz, M.A. 1985. Forelimb anatomy of New World monkeys: myology and the interpretation of primitive anthropoid models. American Journal of Physical Anthropology 68:499-517.

Gambaryan, P. P., Kuznetsov, A. N., Panyutina, A.A., and Gerasimov, S. V. 2015. Shoulder girdle and forelimb myology of extant Monotremata. Russian Journal of Teriology 14:1-56.

Gruber, W. 1868. Uber die varietaten des musculus brachioradialis. Bulletin de l'Academie Imperiale des Sciences de Saint-Pétersbourg 12:277-287.

Gümüşalan, Y., Kalaycıoğlu, A., Yazar, F., Arifoğlu, Y., and Sinav, A. 1997. Accessory extensor carpi radialis muscle and interconnecting muscular bundle. Acta Anatomica 159:57-60.

Haines, R.W. 1939. A revision of the extensor muscles of the forearm in tetrapods. Journal of Anatomy 73:211-233.

Hill, W. C. O. 1960. Primates Comparative Anatomy and Taxonomy: Cebidae. Edinburgh University Press, London.

Hong, M.K., and Hong, M. K. 2005. An uncommon form of the rare extensor carpi radialis accessorius. Annals of Anatomy 187:89-92.

Jain, A., Kumar, R., Kaur, P., and Angel. 2014. Variations in extensor carpi radialis longus and brevis - a cadaveric study. International Journal of Biomedical Research 5 (6):392-395.
Khaledpour, C., and Schindelmeiser, J. 1994. Atypical course of the rare accessory extensor carpi radialis muscle. Journal of Anatomy 184:161-163

LeDouble, A.F.1897. Traité des Variations du Système Musculaire de l'Homme et Leur Signification au Point de Vue de l'Anthropologie Zoologique. Schleicher Freres, Paris.

Levit, G. S., Hossfeld, U., and Olsson, L. 2006. From the "modern synthesis" to cybernetics: Ivan Ivanovich Schmalhausen (1884-1963) and his research program for a synthesis of evolutionary and developmental biology. Journal of Experimental Zoology $B$ 306:89-106.

Maclise, J.1846. On the nomenclature of anatomy (addressed to professors Owen and Grant). Lancet 298-301.

Nayak, S. R., Krishnamurthy, A., Prabhu, L. V., Rai, R., Ranade, A. V., and Madhyastha, S. 2008. Anatomical variation of radial wrist extensor muscles: a study in cadavers. Clinics 63:85-90.

Owen, R. 1846. Report on the archetype and homologies of the vertebrate skeleton. Report of the British Association for the Advancement of Science 169-340.

Owen, R. 1848. On the Archetype and Homologies of the Vertebrate Skeleton. John van Voorst, London.

Owen, R. 1849. On the Nature of Limbs. John van Voorst, London.

Pavlinov, I. Ya. 2012. The contemporary concepts of homology in biology: a theoretical review. Biology Bulletin Reviews 2:36-54.

Rao, M. K., Vollala, V.R., Bhat, S.M., Bolla, S, Samuel, V.P., and Pamidi, N. 2006. Four cases of variations in the forearm extensor musculature in a study of hundred limbs and review of literature. Indian Journal of Plastic Surgery 39:141-147.

Richards, R. 2016. Objectivity and the theory of the archetype; pp. 26-37 in Neiman, S., Galison, P., and Doniger, W. (eds.), What Reason Promises: Essays on Reason, Nature and History. De Gruyter, Berlin.

Rodriguez-Nedenführ, M., Vazquez, T., Parkin, I., Nearn, L., and Sañudo, J.R. 2001. Incidence and morphology of the brachioradialis accessorius muscle. Journal of Anatomy 199:353-355.

Rupke, N.A. 1993. Richard Owen's vertebrate archetype. Isis 84:231-251.

Shetty, P., Sirasanagandla, S.R., and Dsouza, M.R. 2014. Possible entrapment of sensory branch of radial nerve by split brachioradialis tendon:an anatomical variation. International Journal of Health Sciences and Research 4:204-207.

Schön, M.A. 1968. The muscular system of the red howling monkey. Bulletin of the United States National Museum 273:1-185.

Sirena, S. 1871. Recerche sulla miologia del Mycetes fuscus. Giornale di Scienze Naturali ed Economiche di Palermo. Part I. Scienze naturali 7:164-244.

Spinner, R. J., and Spinner, M. 1996. Superficial radial nerve compression at the elbow due to an accessory brachioradialis muscle: a case report. The Journal of Hand Surgery 21:369-372.

Vavilov, N. I. 1922. The law of homologous series in variation. Journal of Genetics 12:47-89.

Westling, C. 1889. Anatomische Untersuchungen über Echidna. Bihang till Kongliga Svenska Vetenskaps-Akademiens Handlingar 15:1-71.

Wood, J. 1867. On human muscular variations in their relation to comparative anatomy. Journal of Anatomy and Physiology 1:44-59.

Wood, V.E. 1988. The extensor carpi radialis intermedius tendon. Journal of Hand Surgery 13:242-245.

Youlatos, D.1999. The schizodactylous grasp of the howling monkey. Zeitschrift für Morphologie und Anthropologie 82:187-198.

Youlatos, D. 2000. Functional anatomy of forelimb muscles in Guianan atelines (Platyrrhini: Primates). Annales des Sciences Naturelles Zoologie et Biologie Animale 21:137-151. 